\title{
Improving the Sensitivity and Dynamic Range of Reagentless Fluorescent Immunosensors by Knowledge-Based Design ${ }^{\dagger}$
}

\author{
Martial Renard ${ }^{\ddagger}$ and Hugues Bedouelle* \\ Unit of Molecular Prevention and Therapy of Human Diseases, CNRS URA 2185, Institut Pasteur, 28 rue Docteur Roux, \\ 75724 Paris Cedex 15, France
}

Received May 26, 2004; Revised Manuscript Received August 24, 2004

\begin{abstract}
The variable fragment $(\mathrm{Fv})$ of an antibody can be transformed into a reagentless fluorescent biosensor by mutating a residue into a cysteine in the neighborhood of the paratope (antigen-binding site) and then coupling an environment-sensitive fluorophore, e.g., $N$-((2-(iodoacetoxy)ethyl)- $N$-methyl)amino7-nitrobenz-2-oxa-1,3-diazole (IANBD ester), to the mutant cysteine. For some residues, named operational, the formation of the conjugate does not affect the affinity of the Fv fragment for the antigen, and the binding of the antigen generates a measurable variation in the fluorescence intensity of the conjugate. We tested if this signal variation could be increased by coupling several molecules of fluorophores to the same molecule of Fv. Seven operational residues have been previously identified in the single-chain Fv (scFv) of monoclonal antibody D1.3 (mAbD1.3), directed against lysozyme. Ten double mutants of scFvD1.3, involving these residues, were constructed and coupled to the IANBD ester. The fluorescence of the double conjugates revealed a transfer of resonance energy between the two identical fluorescent groups. This homotranfer could be more important in the free state of the conjugate than in its antigenbound state and increase its sensitivity for the detection of the antigen by up to 2.9-fold. A poorly sensitive conjugate could be improved by coupling a second molecule of fluorophore to residues located far from the paratope. Mutations altering the affinity of $\mathrm{scFvD} 1.3$ for lysozyme were introduced into one of its fluorescent conjugates. Using a mixture of three mutant derivatives of this unique conjugate, we could titrate lysozyme with precision in a concentration range encompassing 3 orders of magnitude.
\end{abstract}

A signal-transducing receptor generally comprises an extracellular domain that recognizes a specific molecular signal, a transmembrane domain through which the signal is transmitted to the interior of the cell and an intracellular domain through which the signal is transformed and amplified, eventually in the form of second messenger molecules. These three domains are assembled in a single-receptor macromolecule (1). Similarly, a biosensor transforms a specific molecular signal into an electrical signal and comprises several modules: a recognition module, which can be biological or biomimetic; a transduction module, which tranforms the recognition event into a measurable signal; and a module of data evaluation. The recognition and transduction modules are integrated into a compact device. A biosensor can function without additional reagent, provide quantitative analytical information, and follow the concentration of an analyte continuously. Important characteristics of a biosensor are its specificity and selectivity, the sensitivity, linearity and speed of the response, the dynamic range of the measurements, the possibility of calibration and accuracy (2).

$\dagger$ This work was supported by grants from the Fondation pour la Recherche Medicale to M.R. (FRM, FDT20020920140/1) and from the French Ministry of Defense (DGA, 20216/DSP/SREA/F).

* To whom correspondence should be addressed. Telephone: +33 1-45688379. Fax: +33-1-40613533. E-mail: hbedouel@pasteur.fr.

† Present address: "Retrovirus Endogenes", CNRS UMR 8122, Institut Gustave Roussy-PR2, 39 rue Camille Desmoulins, 94805 Villejuif Cedex, France.
In a previous work, we have shown that it is possible to transform any antibody into a reagentless fluorescent immunosensor. The antibody is used in the form of a singlechain variable fragment $(\mathrm{scFv}){ }^{1} \mathrm{~A}$ residue of this fragment is identified in the neighborhood of the antigen in their complex. This residue is changed into a cysteine by sitedirected mutagenesis. A fluorophore is chemically coupled to the mutant cysteine. The binding of the antigen shields the fluorophore from the solvent and can be detected by a change of fluorescence. We have established and validated rules of design to choose the coupling residue, either from the three-dimensional structure of the complex between the antibody and antigen or from mutagenesis data on the complementary determining regions (CDRs) of the antibody, so that the fluorophore does not perturb the affinity for the antigen. The above approach and rules of design have enabled us to construct seven operational conjugates from monoclonal antibody D1.3 (mAbD1.3), which is directed against hen egg-white lysozyme (HEL), and four operational conjugates from antibody mAb4E11, which is directed against the dengue virus $(3,4)$. These two antibodies had average properties of antigen recognition, with dissociation constants equal to $6 \mathrm{nM}$ for $\mathrm{mAbD} 1.3$ and $0.1 \mathrm{nM}$ for mAb4E11.

${ }^{1}$ Abbreviations: Fv, variable fragment; scFv, single-chain Fv; mAbD1.3, monoclonal antibody D1.3; HEL, hen egg-white lysozyme; IANBD ester, $N$-((2-(iodoacetoxy)ethyl)- $N$-methyl)amino-7-nitrobenz2-oxa-1,3-diazole; FU, arbitrary unit of fluorescence intensity. 
Such reagentless fluorescent immunosensors have all of the constitutive characteristics of biosensors. Their recognition module (the $\mathrm{scFv}$ fragment) and transduction module (the fluorophore) are assembled in a single macromolecule. They function without additional reagent. Their fluorescence intensity increases linearly with the concentration of antigen within the dynamic range and enables its quantitative measurement. They are as specific for the antigen as their parental antibody because they have the same affinity. They can function in a complex mixture like serum and thus are selective. The measurement can be continuous because it relies on the reversible thermodynamic equilibrium between the immunosensor, its antigen, and their complex. The dynamic range of the concentration in the antigen can extend up to $80 \%$ of the concentration in the immunosensor, if the latter is saturating, and cover more than 1 order of magnitude $(3,4)$.

We searched for a general approach to improve the sensitivity and dynamic range because these parameters are critical for numerous applications. A priori, both the recognition and transduction modules of a biosensor can be engineered. However, because they are integrated in the same macromolecule, it is essential to understand how the engineering of each of them affects the overall performance of the biosensor. In the present study, we established the theoretical relation between the fluorescence signal and the concentration of analyte, as a function of the physicochemical characteristics of a reagentless fluorescent immunosensor. This relation enabled us to precisely define the sensitivity of an immunosensor and determine the parameters on which it depends. We attempted to capitalize on the additivity of the fluorescence intensities to improve the sensitivity of our immunosensors. We introduced two Cys residues into the scFvD1.3 fragment by mutagenesis and chemically coupled two fluorophore groups on each of these mutant scFvD1.3 molecules. The characterization of the conjugates that we obtained from 10 double mutants revealed fluorescence resonance energy homotransfers (FREHTs) between the two identical fluorescent groups (5). The FREHT phenomenon was more important in the free state of the conjugates than in their complex with lysozyme. This differential FREHT could result in a 3 -fold improvement of the conjugate sensitivity.

The dynamic range of an immunoassay has rarely been engineered to our knowledge. The surface concentration on the solid support is generally the only parameter that can be adjusted. The affinity of the antibody cannot be exploited, because a low affinity for the antigen results in the dissociation of their complex during the washing steps. In contrast, a reagentless fluorescent immunosensor functions at equilibrium and without any washing step. Therefore, such an immunosensor should be able to dose its antigens at concentrations higher than its own. To test this hypothesis, we introduced additional mutations into a scFvD1.3 conjugate and thereby modulated its affinity for lysozyme. The fluorescence properties of the conjugate were not affected by the additional mutation, and the effects of the latter on affinity were the same in the context of the parental scFvD1.3 fragment as in the context of the conjugate. Thus, it was possible to engineer the transduction and binding properties of the conjugate independently. A mixture of three mutant derivatives of the same scFvD1.3 conjugate, with different affinities for lysozyme, enabled us to dose it precisely over a dynamic range covering 3 orders of magnitude.

\section{THEORY}

Equilibrium between the Conjugate and Antigen. Let B be a biosensor; A, its antigen; and $\mathrm{C}$, their complex. They associate according to the reaction

$$
\mathrm{A}+\mathrm{B} \leftrightarrow \mathrm{C}
$$

The law of mass conservation gives

$$
[\mathrm{A}]_{0}=[\mathrm{A}]+[\mathrm{C}] \quad[\mathrm{B}]_{0}=[\mathrm{B}]+[\mathrm{C}]
$$

where $[\mathrm{A}]_{0}$ and $[\mathrm{B}]_{0}$ are the total concentrations of $\mathrm{A}$ and $\mathrm{B}$ in the reaction, respectively.

At equilibrium, the law of mass action gives

$$
K_{\mathrm{D}}=[\mathrm{A}][\mathrm{B}] /[\mathrm{C}]
$$

where $K_{\mathrm{D}}$ is the dissociation constant between $\mathrm{A}$ and $\mathrm{B}$. Combining eqs 1.2 and 1.3 gives

$$
[\mathrm{C}]^{2}-[\mathrm{C}]\left(K_{\mathrm{D}}+[\mathrm{A}]_{0}+[\mathrm{B}]_{0}\right)+[\mathrm{A}]_{0}[\mathrm{~B}]_{0}=0
$$

A biosensor with a weak affinity for its antigen can be used at a concentration $[\mathrm{B}]_{0}$ much lower than its $K_{\mathrm{D}}$. In this condition, one successively deduces from eq $1.2,[\mathrm{~B}]<[\mathrm{B}]_{0}$ $\ll K_{\mathrm{D}}$; from eq $1.3,[\mathrm{C}] \ll[\mathrm{A}]$; from eq $1.2,[\mathrm{~A}] \approx[\mathrm{A}]_{0}$; and from eqs $1.2-1.3$

$$
[\mathrm{C}] \approx[\mathrm{A}]_{0}[\mathrm{~B}]_{0} /\left(K_{\mathrm{D}}+[\mathrm{A}]_{0}\right)
$$

which is the equation for the saturation of B by A.

Fluorescence of the Conjugates. In a previous work (4), we have established that if $F$ is the global fluorescence intensity of a biosensor in a reaction mixture; $F_{0}$ and $F_{\infty}$ are its signals at zero and saturating concentrations of antigen; $f_{\mathrm{a}}, f_{\mathrm{b}}$, and $f_{\mathrm{c}}$, are the molar fluorescence intensities of the antigen, free biosensor, and complex between the biosensor and antigen, respectively, then

$$
\begin{gathered}
F_{0}=f_{\mathrm{b}}[\mathrm{B}]_{0} \\
F_{\infty}=\left(f_{\mathrm{c}}-f_{\mathrm{a}}\right)[\mathrm{B}]_{0} \\
\left(F-F_{0}\right) / F_{0}=\left(\left(F_{\infty}-F_{0}\right) / F_{0}\right)\left([\mathrm{C}] /[\mathrm{B}]_{0}\right)
\end{gathered}
$$

with the notations of the first paragraph of this section. In the following, we note $\Delta F=F-F_{0}, \Delta F_{\infty}=F_{\infty}-F_{0}$, and $\Delta f_{\mathrm{c}}=f_{\mathrm{c}}-f_{\mathrm{a}}-f_{\mathrm{b}}$. Then

$$
F=F_{0}+\Delta f_{\mathrm{c}}[\mathrm{C}]
$$

Because the value of $f_{\mathrm{a}}$ is usually negligible at the wavelength of the measurements, $\Delta f_{\mathrm{c}} \approx f_{\mathrm{c}}-f_{\mathrm{b}}$.

Sensitivity of the Conjugates and Lower Limit of Detection. Because $[\mathrm{C}]$ is a function of $[\mathrm{A}]_{0}$, it can be developed into 
a Taylor series in the neighborhood of $[\mathrm{A}]_{0}=0$. In these conditions, an approximation of eq 2.3 is given by

$$
\Delta F / F_{0} \approx\left(\Delta F_{\infty} / F_{0}\right)\left(\mathrm{d}[\mathrm{C}] / \mathrm{d}[\mathrm{A}]_{0}\right)_{[\mathrm{A}]_{0}=0}\left([\mathrm{~A}]_{0} /[\mathrm{B}]_{0}\right)
$$

The differentiation of eq 1.4 gives

$$
\begin{array}{r}
\left(2[\mathrm{C}]-K_{\mathrm{D}}-[\mathrm{A}]_{0}-[\mathrm{B}]_{0}\right) \mathrm{d}[\mathrm{C}]+\left([\mathrm{B}]_{0}-\right. \\
[\mathrm{C}]) \mathrm{d}[\mathrm{A}]_{0}=0
\end{array}
$$

For $[\mathrm{A}]_{0}=0$, which implies $[\mathrm{C}]=0$, eq 3.2 gives

$$
\left(\mathrm{d}[\mathrm{C}] / \mathrm{d}[\mathrm{A}]_{0}\right)_{[\mathrm{A}]_{0}=0}=\left(1+\left(K_{\mathrm{D}} /[\mathrm{B}]_{0}\right)\right)^{-1}
$$

Combining eqs 3.1 and 3.3 gives, for the low values of $[\mathrm{A}]_{0}$

$$
\Delta F / F_{0} \approx\left(\Delta F_{\infty} / F_{0}\right)\left(1+\left(K_{\mathrm{D}} /[\mathrm{B}]_{0}\right)\right)^{-1}\left([\mathrm{~A}]_{0} /[\mathrm{B}]_{0}\right)
$$

Combining eqs 2.1, 2.2, and 3.4 gives, for the low values of $[\mathrm{A}]_{0}$

$$
\Delta F \approx \Delta f_{\mathrm{c}}\left(1+\left(K_{\mathrm{D}} /[\mathrm{B}]_{0}\right)\right)^{-1}[\mathrm{~A}]_{0}
$$

The sensitivity $s$, relating $\Delta F$ and $[\mathrm{A}]_{0}$, the relative sensitivity $s_{\mathrm{r}}$, relating $\Delta F / F_{0}$ and $[\mathrm{A}]_{0} /[\mathrm{B}]_{0}$, and the lower limit of detection of the biosensor can be deduced from eqs 3.4 and 3.5

$$
\begin{gathered}
s=\Delta f_{\mathrm{c}}\left(1+\left(K_{\mathrm{D}} /[\mathrm{B}]_{0}\right)\right)^{-1} \\
s_{\mathrm{r}}=\left(\Delta F_{\infty} / F_{0}\right)\left(1+\left(K_{\mathrm{D}} /[\mathrm{B}]_{0}\right)\right)^{-1} \\
{[\mathrm{~A}]_{0} \approx\left(1+\left(K_{\mathrm{D}} /[\mathrm{B}]_{0}\right)\right)\left(\Delta F / \Delta f_{\mathrm{c}}\right)}
\end{gathered}
$$

When $K_{\mathrm{D}} \ll[\mathrm{B}]_{0}$, eqs $3.6-3.8$ simplify into

$$
s \approx \Delta f_{\mathrm{c}} \quad s_{\mathrm{r}} \approx \Delta F_{\mathrm{\infty}} / F_{0}=\Delta f_{\mathrm{c}} / f_{\mathrm{b}} \quad[\mathrm{A}]_{0} \approx \Delta F / \Delta f_{\mathrm{c}}
$$

The value of $s$ depends on the spectrofluorometer and its setup. This dependency did not interfere with our comparison of various conjugates because all of our measurements were done with the same spectrofluorometer, setup of the instrument, and rhodamine 101 as the internal standard. However, $s$ does not enable one to compare the performances of conjugates between different laboratories. In contrast, the values of $s_{\mathrm{r}}$ and of the lower limit of detection $\delta[\mathrm{A}]_{0}$ are independent of the spectrofluorometer and of its setup. Equations 3.7 and 3.9 justify our use of the ratio $\Delta F_{\infty} / F_{0}$ to compare conjugates in our previous studies $(3,4)$.

Mixture of Immunosensors. The affinity of one scFvD1.3 conjugate for lysozyme was engineered by mutations. Three biosensors $\mathrm{B}_{i}, i=1,2$, or 3 , which derived from this same conjugate and had $K_{\mathrm{D}_{i}}$ values as respective dissociation constants, were mixed in equimolar amounts and used to titrate antigen $\mathrm{A}$. In such conditions, eqs 1.1 and 1.3 hold for each biosensor $\mathrm{B}_{i}$ and eq 1.2 is replaced by

$$
\begin{array}{r}
{[\mathrm{A}]_{0}=[\mathrm{A}]+\sum\left[\mathrm{C}_{i}\right] \text { and }\left[\mathrm{B}_{i}\right]_{0}=\left[\mathrm{B}_{i}\right]+\left[\mathrm{C}_{i}\right]=\text { constant }} \\
i=1,2 \text { or } 3(4.1)
\end{array}
$$

where $\left[\mathrm{C}_{i}\right]$ is the concentration of the complex between $\mathrm{B}_{i}$ and $\mathrm{A}$. The solution of this set of equations is given by an

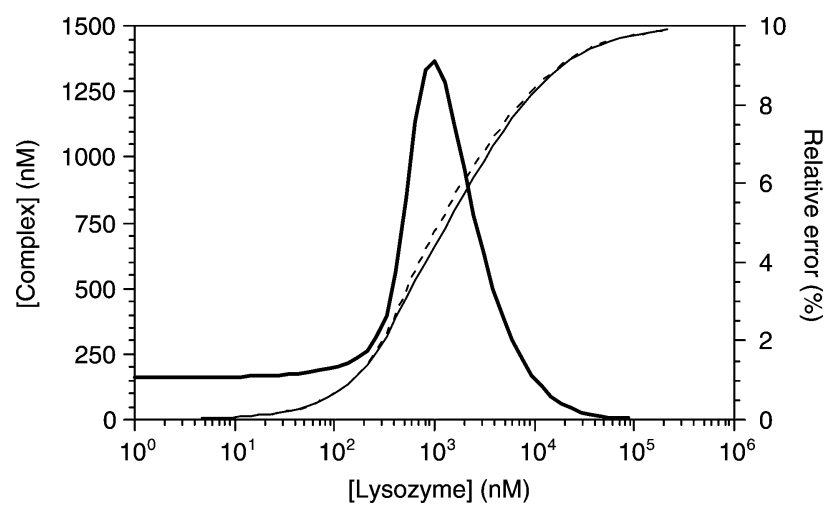

FIGURE 1: Numerical simulation of the interaction between three mutant derivatives of an antibody and its antigen. The dissociation constants $K_{\mathrm{D}_{1}}, K_{\mathrm{D}_{2}}$, and $K_{\mathrm{D}_{3}}$ of the three mutant derivatives were equal to 8,830 , and $6100 \mathrm{nM}$, respectively. The total concentration of the complex $\sum\left[\mathrm{C}_{i}\right], i=1,2$, and 3 , was calculated as a function of the total concentration in the antigen $[\mathrm{A}]_{0}$ according to two different models. The full model assumed that the three antibody derivatives were simultaneously in equilibrium with their common antigen (thin line). The simplified model assumed that the highaffinity derivative formed a complex with the antigen independently of the two low-affinity derivatives (dotted line). The total concentration $\left[\mathrm{B}_{i}\right]_{0}$ of each antibody derivative was equal to $500 \mathrm{nM}$. The difference in $\sum\left[\mathrm{C}_{i}\right]$ when going from the full model to the simplified model was compared to the value of $\Sigma\left[C_{i}\right]$ in the full model (relative error; thick line).

equation of the fourth power and is difficult to fit to the experimental data. We therefore assumed that, if $K_{1} \ll\left[\mathrm{B}_{i}\right]_{0}$ $\ll K_{2}<K_{3}$ for $i=1,2$, and 3, then $\mathrm{B}_{1}$ binds A independently of $\mathrm{B}_{2}$ and $\mathrm{B}_{3}$. Equations 1.4 and 1.5 then give

$$
\begin{aligned}
{\left[\mathrm{C}_{1}\right]^{2}-\left[\mathrm{C}_{1}\right]\left(K_{\mathrm{D}_{1}}+[\mathrm{A}]_{0}+\left[\mathrm{B}_{1}\right]_{0}\right)+[\mathrm{A}]_{0}\left[\mathrm{~B}_{1}\right]_{0}=0 } \\
{\left[\mathrm{C}_{2}\right] \approx[\mathrm{A}]_{\mathrm{r}}\left[\mathrm{B}_{2}\right]_{0} /\left(K_{\mathrm{D}_{2}}+[\mathrm{A}]_{\mathrm{r}}\right) } \\
{\left[\mathrm{C}_{3}\right] \approx[\mathrm{A}]_{\mathrm{r}}\left[\mathrm{B}_{3}\right]_{0} /\left(K_{\mathrm{D}_{3}}+[\mathrm{A}]_{\mathrm{r}}\right) }
\end{aligned}
$$

where $[\mathrm{A}]_{\mathrm{r}}=[\mathrm{A}]_{0}-\left[\mathrm{C}_{1}\right]=[\mathrm{A}]+\left[\mathrm{C}_{2}\right]+\left[\mathrm{C}_{3}\right]$. We checked that the above assumption was valid by comparing numerical solutions of the exact and approximate (4.2-4.4) sets of equations (Figure 1). We found experimentally that the three biosensors, which we derived from the same conjugate, had identical values for their molar intensities of fluorescence $f_{\mathrm{b}}$ and $f_{\mathrm{c}}$ (see the Results). In these conditions, eqs 2.1 and 2.4 give

$$
\begin{array}{r}
F=\sum\left(f_{\mathrm{b}}\left[\mathrm{B}_{i}\right]_{0}+\Delta f_{\mathrm{c}}\left[\mathrm{C}_{i}\right]\right)=F_{0}+\Delta f_{\mathrm{c}}\left(\sum\left[\mathrm{C}_{i}\right]\right) \\
i=1,2, \text { and } 3
\end{array}
$$

where $F_{0}$ is the signal of the mixture of biosensors, each at a concentration $\left[\mathrm{B}_{i}\right]_{0}$ and in its free state.

Titration of Immunosensors by Successive Additions of Antigen. A biosensor, at an initial concentration $[\mathrm{B}]_{1}$ in an initial volume $V_{1}$, was titrated by addition of increasing volumes $v$ from a stock solution of antigen at concentration $[\mathrm{A}]_{1}$. In general, volume $v$ resulted from successive additions of small aliquots. The law of mass conservation gives

$$
\left(V_{1}+v\right)[\mathrm{B}]_{0}=V_{1}[\mathrm{~B}]_{1} \quad\left(V_{1}+v\right)[\mathrm{A}]_{0}=v[\mathrm{~A}]_{1}
$$

If we note that $a=[\mathrm{B}]_{1} /[\mathrm{A}]_{1}$, then $[\mathrm{B}]_{0}=[\mathrm{B}]_{1}-a[\mathrm{~A}]_{0}$, and eqs 1.4 and 1.5 can be rearranged as 


$$
\begin{array}{r}
{[\mathrm{C}]^{2}-[\mathrm{C}]\left(K_{\mathrm{D}}+(1-a)[\mathrm{A}]_{0}+[\mathrm{B}]_{1}\right)+[\mathrm{A}]_{0}\left([\mathrm{~B}]_{1}-\right.} \\
\left.a[\mathrm{~A}]_{0}\right)=0 \\
{[\mathrm{C}] \approx[\mathrm{A}]_{0}\left([\mathrm{~B}]_{1}-a[\mathrm{~A}]_{0}\right) /\left(K_{\mathrm{D}}+[\mathrm{A}]_{0}\right)}
\end{array}
$$

If $[\mathrm{A}]_{0} \ll[\mathrm{A}]_{1}$ for every value of $[\mathrm{A}]_{0}$ in the experiment or equivalently if $v \ll V_{1}$ for every value of $v$, eq 5.3 simplifies into

$$
[\mathrm{C}] \approx[\mathrm{A}]_{0}[\mathrm{~B}]_{1} /\left(K_{\mathrm{D}}+[\mathrm{A}]_{0}\right)
$$

If in addition $[\mathrm{B}]_{1} \ll[\mathrm{A}]_{1}$, eq 5.2 simplifies into

$$
[\mathrm{C}]^{2}-[\mathrm{C}]\left(K_{\mathrm{D}}+[\mathrm{A}]_{0}+[\mathrm{B}]_{1}\right)+[\mathrm{A}]_{0}[\mathrm{~B}]_{1}=0
$$

A comparison between eqs 5.4 and 1.5 and between eqs 5.5 and 1.4 shows that the difference between $[\mathrm{B}]_{0}$ and $[\mathrm{B}]_{1}$ can be neglected if the above conditions are satisfied. However, $[\mathrm{A}]_{0}$ has to be calculated from eq 5.1 if eqs $5.2-5.5$ are applied. A similar reasoning can be applied to eqs 4.2-4.4.

\section{MATERIALS AND METHODS}

Construction and Production of the Conjugates. Buffer A contained $50 \mathrm{mM}$ Tris- $\mathrm{HCl}(\mathrm{pH} 7.5)$ and $150 \mathrm{mM} \mathrm{NaCl}$. Phagemid pMR1, coding for the scFvD1.3 fragment has been described (3). The fluorophore $N$-((2-(iodoacetoxy)ethyl)$\mathrm{N}$-methyl)amino-7-nitrobenz-2-oxa-1,3-diazole (IANBD ester) was purchased from Molecular Probes. Mutations were constructed by the Kunkel's method, with the single-stranded DNA of pMR1 or mutant derivatives of pMR1 as the template (6). The mutant scFvs were produced in the periplasmic space of Escherichia coli, purified, and coupled with the fluorophore as described (3). The yield of coupling between the fluorophore and the scFv mutants was calculated from their absorbance spectra as described, with $\epsilon_{280^{-}}$ $(\mathrm{scFvD} 1.3)=51.13 \mathrm{mM}^{-1} \mathrm{~cm}^{-1}, \epsilon_{280}(-\mathrm{S}-\mathrm{ANBD})=2.1$ $\mathrm{mM}^{-1} \mathrm{~cm}^{-1}$, and $\epsilon_{500}\left(-\mathrm{S}\right.$-ANBD) $=31.6 \mathrm{mM}^{-1} \mathrm{~cm}^{-1}$ (3).

Molar Fluorescences of the Conjugates. The fluorescences of the IANBD ester group and of its conjugates with the scFvD1.3 fragment were recorded with a Perkin-Elmer LS5B spectrofluorometer and rhodamine 101 as an internal reference. The wavelength of the excitation light was equal to $480 \mathrm{~nm}$, and the emitted light was measured at $535 \mathrm{~nm}$. The conjugates were used at concentrations lower than 500 $\mathrm{nM}$ to minimize the inner-filter effect $\left(A_{500 \mathrm{~nm}}<0.016\right)$, either in the absence or in the presence of a saturating concentration of antigen, i.e., HEL ( $20 \mu \mathrm{M}$, Sigma). The fluorescence intensity of the conjugates was obtained as the difference between those of the sample and the buffer alone. We checked that the molar intensity of fluorescence $f_{\mathrm{a}}$ for the antigen alone was negligible under our experimental conditions. The molar intensity of fluorescence $f_{\mathrm{b}}$ for the single conjugates was calculated as the ratio between the intensity of the light emitted by the conjugate and the concentration of coupled fluorophore. When the value of $f_{\mathrm{b}}$ was calculated in this way, it was not affected by the residual concentration of noncoupled $\mathrm{scFv}$ molecules in the preparation of the conjugate and by the small variations of coupling yield between different preparations. The yield of coupling between the fluorophore and the double Cys mutants of the $\mathrm{scFv}$ fragment was always close to two molecules of fluorophore per molecule of $\mathrm{scFv}$. We therefore considered that the two mutant Cys residues were coupled with the same efficiency. The value of $f_{\mathrm{b}}$ for the double conjugates was calculated as twice the ratio between the intensity of the emitted light and the concentration of coupled fluorophore.

Titration of the Conjugates with Lysozyme. We constructed three scFvD1.3 conjugates, with the same properties of fluorescence and different affinities, by coupling IANBD ester to residue L-Thr94 and introducing the side-chain Tyr (wild type), Phe, or Ala in residue position $\mathrm{H}-101$ by mutagenesis. The fluorescence intensities of the three conjugates, taken individually $(500 \mathrm{nM}, 1 \mathrm{~mL})$ or in equimolar amounts $(500 \mathrm{nM}$ each, $1 \mathrm{~mL})$, were measured after successive additions of aliquots $(2 \mu \mathrm{L})$ from a fresh concentrated solution of lysozyme. The concentration of lysozyme was measured using $\epsilon_{280}=37.97 \mathrm{mM}^{-1} \mathrm{~cm}^{-1}$ (7). Repeated measurements at a saturating concentration of lysozyme showed that the fluorophore was not photobleached during the experiment. The equation linking the fluorescence intensity of the reaction mixture to the total concentration of lysozyme in this mixture was fitted to the experimental measurements with the pro Fit 5.0 software (Cherwell Scientific Publishing). The total concentration of the parental scFvD1.3(L-T94ANBD) conjugate was higher than its $K_{\mathrm{D}}$ for lysozyme $(8 \mathrm{nM})$, and therefore, we used eqs 5.2 and 2.4 , with $F_{0}, \Delta f_{\mathrm{c}},[\mathrm{B}]_{1}$, and $K_{\mathrm{D}}$ as fitting parameters. The total concentrations of the scFvD1.3(L-T94ANBD) derivatives, having altered affinities for lysozyme, were lower than their $K_{\mathrm{D}}$ values $(830$ and $6100 \mathrm{nM}$ for the H-Y101F and H-Y101A mutants, respectively), and therefore, we used eqs 5.3 and 2.4 , with $F_{0}, \Delta f_{\mathrm{c}}$, and $K_{\mathrm{D}}$ as fitting parameters. For the titration of an equimolar mixture of the three conjugates by successive additions of antigen, we used derivatives of eqs 4.2-4.4, obtained as described in the last paragraph of the Theory, and eq 4.5 with $F_{0}, \Delta f_{\mathrm{c}}, K_{\mathrm{D}_{\mathrm{i}}}$, and the common value of $\left[\mathrm{B}_{i}\right]_{1}, i=1,2$, and 3 , as fitting parameters.

Analysis of Errors and Sensitivity of the Conjugates. The differentiation of eq 4.5 gives

$$
\begin{array}{r}
\mathrm{d} F=\Delta f_{\mathrm{c}}\left(\sum \mathrm{d}\left[\mathrm{C}_{i}\right]\right)=\Delta f_{\mathrm{c}}\left(\sum \mathrm{d}\left[\mathrm{C}_{i}\right] / \mathrm{d}[\mathrm{A}]_{0}\right) \mathrm{d}[\mathrm{A}]_{0} \\
\text { with } i=1,2, \text { and } 3
\end{array}
$$

Therefore, the relative error on the concentration $[\mathrm{A}]_{0}$ of the antigen is given by

$$
\mathrm{d}[\mathrm{A}]_{0} /[\mathrm{A}]_{0}=\left(\mathrm{d} F / \Delta f_{\mathrm{c}}\right)\left(\sum \mathrm{d}\left[\mathrm{C}_{\mathrm{i}}\right] / \mathrm{d}[\mathrm{A}]_{0}\right)^{-1}[\mathrm{~A}]_{0}{ }^{-1}
$$

The ratio $d[\mathrm{~A}]_{0} /[\mathrm{A}]_{0}$ was calculated from eq 6.2 and the routines Solve, D, and Table of the Mathematica software (8). We used the dissociation constants and molar fluorescences of the three individual conjugates in these calculations. The error $\delta F$ on the fluorescence intensity was estimated from the standard deviation between the values of $F$, measured experimentally and calculated from the fitted equation, in experiments of titration by individual conjugates (3). It was equal to $2.0 \pm 0.1 \mathrm{FU}$ [mean \pm standard error (SE)], and therefore, we took $\delta F=2.0 \mathrm{FU}$ for the calculation of the relative errors and lower limits of detection.

\section{RESULTS}

Molar Fluorescences of Single Conjugates. The fluorescence intensity $F$ of the conjugates $(500 \mathrm{nM})$ between the 


\begin{tabular}{|c|c|c|c|c|}
\hline residue & $f_{\mathrm{b}}\left(\mathrm{FU} \mu \mathrm{M}^{-1}\right)$ & $f_{\mathrm{c}}\left(\mathrm{FU} \mu \mathrm{M}^{-1}\right)$ & $\Delta f_{\mathrm{c}}\left(\mathrm{FU} \mu \mathrm{M}^{-1}\right)$ & $\Delta f_{\mathrm{c}} / f_{\mathrm{b}}(\%)$ \\
\hline L-Asn 31 & $421 \pm 24$ & $467 \pm 31$ & $46 \pm 4$ & $11 \pm 1$ \\
\hline L-Tyr49 & $261 \pm 14$ & $550 \pm 21$ & $289 \pm 19$ & $111 \pm 10$ \\
\hline L-Thr52 & $247 \pm 57$ & $274 \pm 59$ & $27 \pm 9$ & $11 \pm 5$ \\
\hline L-Thr53 & $633 \pm 34$ & $723 \pm 37$ & $90 \pm 7$ & $14 \pm 1$ \\
\hline L-Ser65 & $327 \pm 5$ & $319 \pm 4$ & $8 \pm 1$ & $-2 \pm 1$ \\
\hline L-Thr69 & $284 \pm 3$ & $280 \pm 3$ & $4 \pm 1$ & $-1 \pm 1$ \\
\hline L-Ser93 & $113 \pm 6$ & $216 \pm 10$ & $103 \pm 7$ & $91 \pm 8$ \\
\hline L-Thr94 & $441 \pm 34$ & $813 \pm 74$ & $372 \pm 44$ & $84 \pm 12$ \\
\hline H-Tyr32 & $244 \pm 2$ & $315 \pm 3$ & $71 \pm 1$ & $29 \pm 1$ \\
\hline
\end{tabular}

${ }^{a}$ Column 1, residue of scFvD1.3 that was changed into Cys and coupled with IANBD ester; columns $2-5$, see the Theory for the definitions of $f_{\mathrm{b}}, f_{\mathrm{c}}, \Delta f_{\mathrm{c}}$, and $\Delta f_{\mathrm{c}} / f_{\mathrm{b}}$. FU, fluorescence units. Mean $\pm \mathrm{SE}$ for two independent preparations of the conjugate.

scFvD1.3 fragment and the IANBD ester fluorophore, constructed from single Cys mutants, was measured before and after the addition of lysozyme at a saturating concentration $(20 \mu \mathrm{M})$. The parameters that describe the fluorescence properties of the conjugates are reported in Table 1, where the molar intensities of fluorescence are $f_{\mathrm{b}}$ for the free state of the conjugate and $f_{\mathrm{c}}$ for its bound state, the variation of molar intensity on antigen binding is $\Delta f_{\mathrm{c}}$, and the relative variation of fluorescence intensity on antigen binding is $\Delta F_{\infty}$ / $F_{0}=\Delta f_{\mathrm{c}} / f_{\mathrm{b}}$ (see definitions in the Theory). The $\Delta f_{\mathrm{c}}$ and $\Delta f_{\mathrm{c}} l$ $f_{\mathrm{b}}$ parameters are related respectively to the sensitivity $s$ and relative sensitivity $s_{\mathrm{r}}$ of the conjugates by eqs $3.6-3.9$. The values of $f_{\mathrm{b}}$ and $f_{\mathrm{c}}$ varied between different conjugates, 5.6and 3.7-fold, respectively. These variations suggested that the accessibility of the fluorescent group to the solvent varied widely with its coupling site, as observed for another antibody (4). The values of $\Delta f_{\mathrm{c}}$ and $\Delta f_{\mathrm{c}} / f_{\mathrm{b}}$ were the highest for the three conjugates that we constructed from residues L-Tyr49, L-Ser93, and L-Thr94. They were close to zero for those constructed from L-Ser65 and L-Thr69, as expected from the positions of the two residues in the crystal structure of the complex between the FvD1.3 fragment and lysozyme, i.e., far from lysozyme (Figure 2) (9). We estimated that $\delta F$, the error that was made on the value of $F$ with our experimental setup, was equal to $2.0 \mathrm{FU}$ (see the Materials and Methods). Using this value of $\delta F$ and eq 3.9, we calculated that the lowest limit of detection was obtained with the scFvD1.3(L-T94ANBD) conjugate and equal to 5.4 nM lysozyme (1.4 ppm).

Design and Production of Double Conjugates. A total of 10 double conjugates between the scFvD1.3 fragment and the IANBD ester fluorophore was constructed to analyze

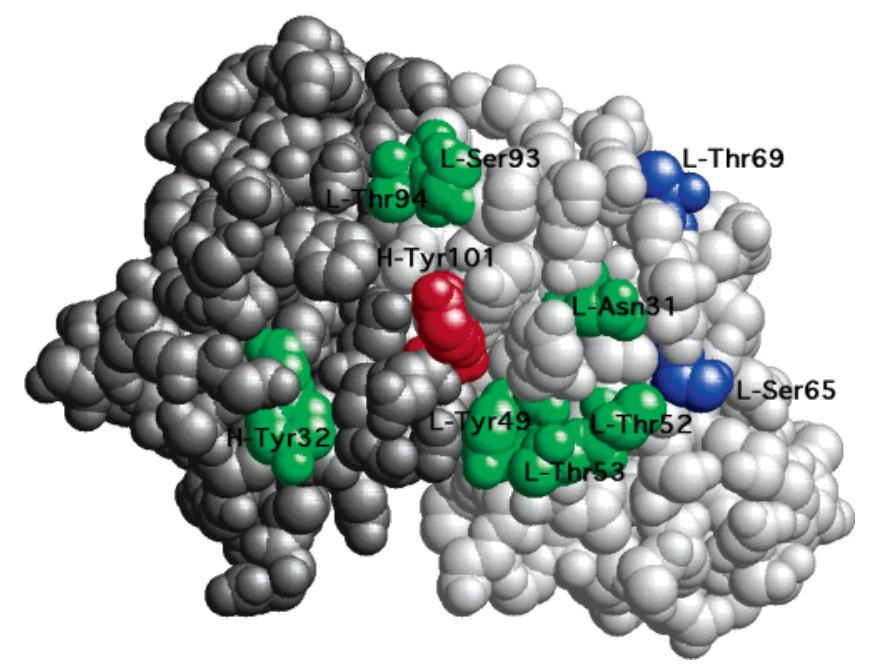

FIGURE 2: Positions of the coupling sites in the structure of FvD1.3. The FvD1.3 fragment is seen from the position of lysozyme in their complex (9). Light gray, $\mathrm{V}_{\mathrm{L}}$; medium gray, $\mathrm{V}_{\mathrm{H}}$; green, operational residues used for fluorophore coupling (3); blue, nonoperational residues used for the coupling of a second fluorophore; red, residue $\mathrm{H}-\mathrm{Ty}$ 101, which is located in the center of the paratope. The figure was drawn with Rasmol version 2.7 (24).

how the properties of the single conjugates would combine, whether the two fluorophores would interact with each other at the surface of one scFvD1.3 molecule, and whether the relative position of the two coupling residues was important (Figure 2). We chose couples of target residues that were distant in the same variable domain (lines 1-3 of Table 2), close (lines 4-6 of Table 2), or distributed between the two variable domains $\mathrm{V}_{\mathrm{H}}$ and $\mathrm{V}_{\mathrm{L}}$ (line 7 of Table 2). We combined the two coupling sites that gave the highest values of $\Delta f_{\mathrm{c}}$ (line 8 of Table 2) and one site that gave a weak value of $\Delta f_{c}$, with sites that were distant from the paratope of the antibody (i.e., its antigen-binding site; see lines 9 and 10 of Table 2). The 10 double Cys mutants of scFvD1.3 were produced in the periplasm of E. coli and a soluble form, and they were purified through their hexahistidine tag in the same conditions as the single mutants. The yields of production were slightly lower for the double mutants than for the single mutants on average.

The double Cys mutants of scFvD1.3 were coupled with IANBD ester by the same method as the single mutants. This method included a mild reduction with 2-mercaptoethanol to reactivate the thiol groups and then their reaction with the IANBD ester. The concentrations of protein and fluorophore in the purified preparations of conjugates were

Table 2: Fluorescence Parameters of the Double Conjugates ${ }^{a}$

\begin{tabular}{|c|c|c|c|c|c|}
\hline residues & distance $(\AA)$ & $f_{1+2, \mathrm{~b}}\left(\mathrm{FU} \mu \mathrm{M}^{-1}\right)$ & $f_{1+2, \mathrm{c}}\left(\mathrm{FU} \mu \mathrm{M}^{-1}\right)$ & $e_{\mathrm{b}}(\%)$ & $e_{\mathrm{c}}(\%)$ \\
\hline L-Ser93 + L-Thr52 & 21.7 & $252 \pm 8$ & $366 \pm 2$ & $30 \pm 3$ & $25 \pm 6$ \\
\hline L-Ser93 + L-Thr53 & 22.0 & $332 \pm 22$ & $504 \pm 20$ & $56 \pm 7$ & $47 \pm 5$ \\
\hline L-Asn31 + L-Thr53 & 11.3 & $572 \pm 14$ & $740 \pm 20$ & $46 \pm 5$ & $38 \pm 4$ \\
\hline L-Asn31 + L-Thr52 & 6.6 & $244 \pm 12$ & $295 \pm 32$ & $64 \pm 16$ & $60 \pm 17$ \\
\hline L-Thr52 + L-Thr53 & 8.1 & $314 \pm 30$ & $386 \pm 4$ & $65 \pm 23$ & $61 \pm 19$ \\
\hline L-Ser93 + L-Thr94 & 5.4 & $114 \pm 6$ & $220 \pm 4$ & $79 \pm 10$ & $79 \pm 9$ \\
\hline L-Ser93 + H-Tyr32 & 19.6 & $332 \pm 22$ & $536 \pm 24$ & $7 \pm 1$ & $-1 \pm 1$ \\
\hline L-Tyr49 + L-Thr94 & 17.0 & $380 \pm 3$ & $790 \pm 22$ & $46 \pm 6$ & $42 \pm 5$ \\
\hline L-Asn31 + L-Ser65 & 10.2 & $667 \pm 16$ & $802 \pm 28$ & $10 \pm 1$ & $-2 \pm 1$ \\
\hline L-Asn31 + L-Thr69 & 8.8 & $578 \pm 40$ & $614 \pm 72$ & $18 \pm 2$ & $17 \pm 3$ \\
\hline
\end{tabular}

${ }^{a}$ Column 1, residues of scFvD1.3 that were changed into Cys and coupled with IANBD ester; column 2, distance between the two $\gamma$ atoms of the residues in column 1 ; columns $3-6$, see the Theory and eq 7 for the definitions of $f_{1+2, \mathrm{~b}}, f_{1+2, \mathrm{c}}, e_{\mathrm{b}}$, and $e_{\mathrm{c}}$. The $1+2$ in lower script refers to the double conjugate. Mean \pm SE for two independent preparations of the conjugate. 
Table 3: Comparison of the Fluorescence Parameters for the Single and Double Conjugates ${ }^{a}$

\begin{tabular}{ccccc}
\hline \multicolumn{1}{c}{ residues } & $\Delta f_{1+2, \mathrm{c}}\left(\mathrm{FU} \mu \mathrm{M}^{-1}\right)$ & gain in $\Delta f_{\mathrm{c}}$ & $\Delta f_{1+2, \mathrm{~d}} / f_{1+2, \mathrm{~b}}(\%)$ & gain in $\Delta f_{\mathrm{c}} / f_{\mathrm{b}}$ \\
\hline L-Ser93 + L-Thr52 & $114 \pm 4$ & $1.11 \pm 0.08$ & $45 \pm 2$ & $0.49 \pm 0.05$ \\
L-Ser93 + L-Thr53 & $172 \pm 13$ & $1.05 \pm 0.11$ & $27 \pm 3$ & $0.30 \pm 0.04$ \\
L-Asn31 + L-Thr53 & $168 \pm 6$ & $1.86 \pm 0.17$ & $30 \pm 1$ & $2.14 \pm 0.17$ \\
L-Asn31 + L-Thr52 & $151 \pm 18$ & $1.11 \pm 0.16$ & $21 \pm 3$ & $1.91 \pm 0.32$ \\
L-Thr52 + L-Thr53 & $72 \pm 7$ & $0.80 \pm 0.10$ & $93 \pm 3$ & $1.64 \pm 0.30$ \\
L-Ser93 + L-Thr94 & $106 \pm 6$ & $1.98 \pm 0.03$ & $61 \pm 6$ & $1.02 \pm 0.17$ \\
L-Ser93 + H-Tyr32 & $204 \pm 16$ & $1.10 \pm 0.13$ & $108 \pm 3$ & $0.67 \pm 0.09$ \\
L-Tyr49 + L-Thr94 & $410 \pm 12$ & $2.94 \pm 0.29$ & $20 \pm 1$ & $1.03 \pm 0.10$ \\
L-Asn31 + L-Ser65 & $135 \pm 6$ & $0.78 \pm 0.13$ & $6 \pm 1$ & $0.43 \pm 0.12$ \\
L-Asn31 + L-Thr69 & $36 \pm 5$ & & $0.43 \pm 0.08$ \\
\hline
\end{tabular}

${ }^{a}$ Column 1, residues of scFvD1.3 that were changed into Cys and coupled with IANBD ester; columns 2 and 4 , see the Theory for the definitions of $\Delta f_{1+2, \mathrm{c}}$ and $\Delta f_{1+2, \mathrm{~d}} / f_{1+2, \mathrm{~b}}$; column 3, gain calculated by $\Delta f_{1+2, \mathrm{c}} / \max \left(\Delta f_{1, \mathrm{c}}, \Delta f_{2, \mathrm{c}}\right)$; column 4 , gain calculated by $\left(\Delta f_{1+2, \mathrm{~d}} / f_{1+2, \mathrm{~b}}\right) / \max \left(\Delta f_{1, \mathrm{~d}} / f_{1, \mathrm{~b}}, \Delta f_{2, \mathrm{~d}}\right.$ $f_{2, \mathrm{~b}}$ ). The 1,2 , and $1+2$ in lower scripts refer to the first single and second single and double conjugates, which were constructed from the residues in column 1. Mean $\pm \mathrm{SE}$ for two independent preparations of the conjugate.

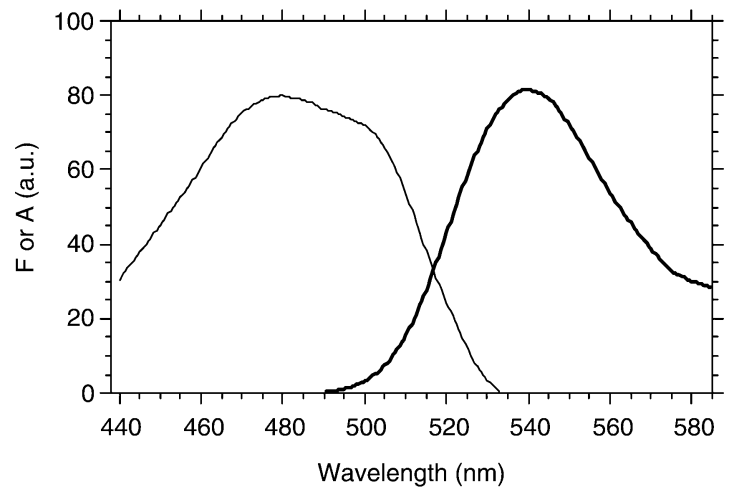

FIGURE 3: Comparison of the excitation (thin line) and emission (thick line) spectra of the -ANBD moiety for a conjugate between 2-mercaptoethanol and IANBD ester. Emission was monitored at $535 \mathrm{~nm}$ for the excitation spectrum, and excitation was at $480 \mathrm{~nm}$ for the emission spectrum. $F$, fluorescence intensity; $A$, absorbance; a.u., arbitrary units. A conjugate between 2-mercaptoethanol and iodoacetamide was used as a blank. The conjugates were obtained by reacting 2 -mercaptoethanol $(10 \mathrm{mM})$ and either IANBD ester $(10 \mu \mathrm{M})$ or iodoacetamide $(10 \mu \mathrm{M})$ in buffer A during $1 \mathrm{~h}$ at room temperature in the dark. The resulting samples were diluted 10fold for the fluorescence measurements.

measured from their absorbance spectra. The different steps resulted in the loss of $60-90 \%$ of the protein material for the single Cys mutants and 80-97\% for the double mutants. These losses suggested that the double Cys mutants aggregated more readily than the single mutants. The coupling yield was equal to $0.74-0.88$ molecule of IANBD per thiol group for the single mutants and equal to $0.72-1.04$ molecules for the double mutants. These yields indicated that the two cysteines of the double mutants were nearly fully coupled to the fluorophore and thus that none of the two thiol groups was preferentially labeled with IANBD ester.

Molar Fluorescences of the Double Conjugates. The molar fluorescences $f_{1+2}$ of the double conjugates were measured in the same conditions as those for the single conjugates $f_{1}$ and $f_{2}$ (Table 2). In general, $f_{1+2}$ was lower than the sum of $f_{1}$ and $f_{2}$ (Tables 1 and 2). This result suggested a fluorescence resonance energy transfer (FRET) between the two identical fluorescent groups that were carried by each molecule of the double conjugate. The relative deviation of the molar fluorescences from additivity was measured by the efficiency of FRET, $e$, between the two fluorescent groups, which is defined by the equation (5)

$$
e=\left(f_{1}+f_{2}-f_{1+2}\right) /\left(f_{1}+f_{2}\right)
$$

The efficiencies of transfer $e_{\mathrm{b}}$ and $e_{\mathrm{c}}$ were calculated for the antigen-free and -bound states of the double conjugates, respectively (Table 2). The value of $e_{\mathrm{b}}$ was higher than zero for all of the double conjugates. Thus, there was a significant FRET between the two identical fluorescent groups in every case. This transfer was possible because the absorption and emission spectra of the -ANBD group overlap and because the distance between the two fluorescent groups, estimated from the positions of the $\gamma$ atoms of the coupling residues (5.4-22 $\AA$ ), was compatible with the Förster distance for the NBD derivatives (Figure 3 and Table 2) (5). The value of $e_{\mathrm{c}}$ was higher than zero for most of the double conjugates, except for those at residues (L-Ser93 and H-Tyr32) and (LAsn31 and L-Ser65). For these two exceptional double conjugates, the value of $e_{\mathrm{c}}$ was equal to zero and showed that the molar fluorescences of the single conjugates added in the corresponding double conjugate, in the antigen-bound state. As a consequence, the efficiency of transfer $e_{\mathrm{c}}$ was lower than $e_{\mathrm{b}}$ for these two exceptional double conjugates; $e_{\mathrm{c}}$ was also marginally lower than $e_{\mathrm{b}}$ for the double conjugate at positions (L-Asn31 and L-Thr53).

Sensitivity of the Double Conjugates. The values of the $\Delta f_{\mathrm{c}}$ and $\Delta f_{\mathrm{c}} / f_{\mathrm{b}}$ parameters for the double conjugates were calculated from their molar fluorescences $f_{\mathrm{b}}$ and $f_{\mathrm{c}}$ and compared with the values for the parental single conjugates (Table 3). The $\Delta f_{\mathrm{c}}$ and $\Delta f_{\mathrm{c}} / f_{\mathrm{b}}$ parameters are related respectively to the sensitivity $s$ and relative sensitivity $s_{\mathrm{r}}$ of the conjugates, as stated above. The gain $s_{1+2} / \max \left(s_{1}, s_{2}\right)$ in sensitivity $s$ between the double conjugate and the best of the two parental single conjugates was small in most cases. However, this gain was significant for 3 double conjugates among the 10 that we studied, with the highest value equal to 2.9 at positions (L-Asn31 and L-Ser65). The gain in relative sensitivity $s_{\mathrm{r}}$ was higher than 1 for 4 double conjugates, with the highest value equal to 2.1 at positions (L-Asn31 and L-Thr53). A gain in $s_{\mathrm{r}}$ was not always paralleled by a gain in $s$, as shown by the double conjugate at positions (L-Asn31 and L-Thr52). Reciprocally, a gain in $s$ was not always paralleled by a gain in $s_{\mathrm{r}}$, as shown by the double conjugate at positions (L-Ser93 and H-Tyr32).

Modulating the Affinity of a Conjugate by Mutations. Among all of the conjugates that we constructed from scFvD1.3, scFvD1.3(L-T94ANBD) had the highest sensitivity. We tested whether we could modulate its affinity by mutations, without altering its fluorescence properties. Mutations $\mathrm{H}-\mathrm{Y} 101 \mathrm{~F}$ and $\mathrm{H}-\mathrm{Y} 101 \mathrm{~A}$ increase the dissociation 


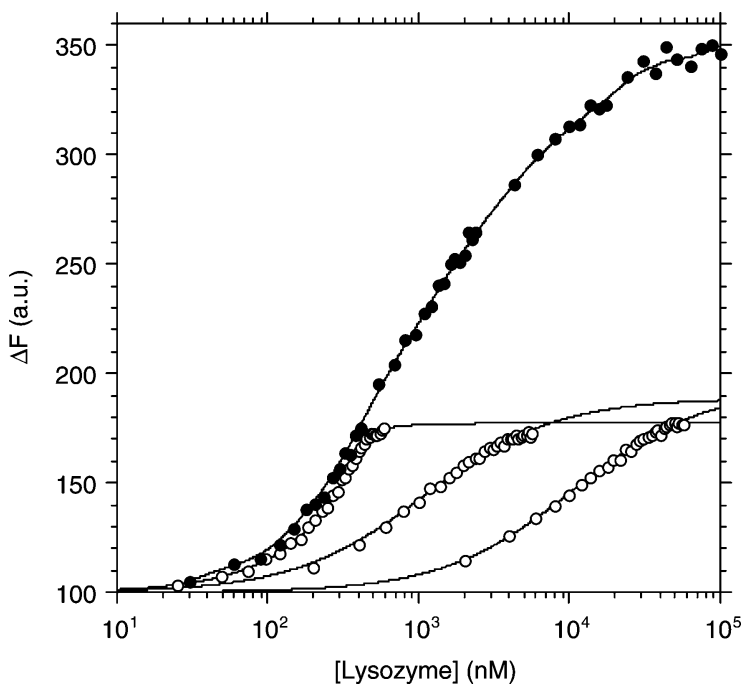

Figure 4: Titration of the scFv(L-T94ANBD) derivatives with lysozyme, either individually or in mixture. The formation of the complexes between the conjugates and lysozyme in buffer A was monitored by the variation of fluorescence intensity $\Delta F=F-F_{0}$ at $535 \mathrm{~nm}$. $\bigcirc$, titration of the conjugates taken individually (500 $\mathrm{nM}$ total concentration); $\boldsymbol{\theta}$, titration of an equimolar mixture of the three conjugates (500 $\mathrm{nM}$ each). The continuous curves correspond to the fitting of eqs 2.4 and 4.5 to the experimental data (see the Materials and Methods).

Table 4: Fluorescence and Affinity Parameters of the scFvD1.3(L-T94ANBD) Derivatives ${ }^{a}$

\begin{tabular}{lcccr}
\hline mutation & $f_{\mathrm{b}}\left(\mathrm{FU} \mathrm{M}^{-1}\right)$ & $f_{\mathrm{c}}\left(\mathrm{FU} \mathrm{M}^{-1}\right)$ & \multicolumn{1}{c}{$K_{\mathrm{D}}(\mathrm{nM})$} & \multicolumn{1}{c}{$K_{\mathrm{D}_{i}}(\mathrm{nM})$} \\
\hline H-Tyr101 & $443 \pm 4$ & $789 \pm 9$ & $8 \pm 2$ & $25 \pm 28$ \\
H-Phe101 & $478 \pm 5$ & $909 \pm 5$ & $830 \pm 30$ & $830 \pm 80$ \\
H-Ala101 & $465 \pm 5$ & $924 \pm 5$ & $6100 \pm 230$ & $6800 \pm 37$ \\
mixture & $460 \pm 2$ & $797 \pm 37$ & na & na \\
\hline
\end{tabular}

${ }^{a}$ Column 1 , residue in position $\mathrm{H}-101$ of the conjugate; columns 2 and 3, see the Theory for the definition of $f_{\mathrm{b}}$ and $f_{\mathrm{c}}$; column $4, K_{\mathrm{D}}$ of each mutant derivative, calculated from a titration reaction containing only that derivative; column $5, K_{\mathrm{D}_{i}}$ of each mutant derivative, calculated from a titration reaction containing an equimolar mixture of the three derivatives; last line, equimolar mixture of the three derivatives; na, not applicable. Mean $\pm \mathrm{SE}$ for two independent preparations of the conjugate.

constant $K_{\mathrm{D}}$ between the FvD1.3 fragment and lysozyme 75and $>850$-fold, respectively $(10-12)$. We introduced them into the scFvD1.3(L-T94C) background. The double mutants of scFvD1.3 were produced, purified, and coupled with IANBD ester in the same conditions as the parental single mutant. The coupling yields ( 84 and $82 \%$, respectively) and the protein yields of the coupling procedure (11 and 16\%, respectively) were similar for the $\mathrm{H}-\mathrm{Y} 101 \mathrm{~F}$ and $\mathrm{H}-\mathrm{Y} 101 \mathrm{~A}$ double mutants as for the parental single mutant ( 97 and $24 \%$, respectively).

The fluorescence intensities of the scFvD1.3(L-T94ANBD) derivatives were measured in the presence of variable concentrations of lysozyme. Equation 2.4, giving the fluorescence intensity of a conjugate as a function of the total concentration in the antigen, was fitted to the experimental values with the characteristic parameters of the conjugate as fitting parameters (see the Materials and Methods and Figure 4). The three conjugates had similar molar intensities of fluorescence $f_{\mathrm{b}}$ and $f_{\mathrm{c}}$ (Table 4). Thus, the mutations at position $\mathrm{H}-101$ did not modify the fluorescence properties of the scFvD1.3(L-T94ANBD) conjugate. We calculated the

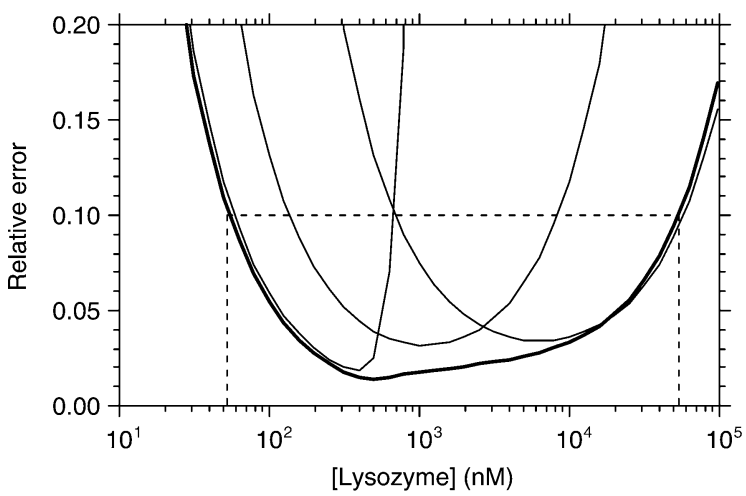

FIGURE 5: Relative error on the concentration of lysozyme in measurements with derivatives of the scFv(L-T94ANBD) conjugate. Thin lines, each derivative of $\mathrm{scFv}(\mathrm{L}-\mathrm{T} 94 \mathrm{ANBD})$ taken individually; thick line, mixture of the three derivatives. The experimental conditions are the same as in Figure 4. The error $\delta F$ on the fluorescence intensity was constant and equal to $2.0 \mathrm{FU}$ (see the Materials and Methods). The dotted lines delineate the range of concentrations in lysozyme that could be measured by the equimolar mixture of the three derivatives with a $10 \%$ accuracy.

free energies of interaction $\Delta G$ between the conjugates and lysozyme from their $K_{\mathrm{D}}$ values (Table 4 ). The variations of $\Delta G$ caused by mutations $\mathrm{H}-\mathrm{Y} 101 \mathrm{~F}$ and $\mathrm{H}-\mathrm{Y} 101 \mathrm{~A}$ were similar in the contexts of the scFvD1.3(L-T94ANBD) conjugate and FvD1.3 variable fragment: $\Delta \Delta G=2.7 \pm$ $0.8 \mathrm{kcal} \mathrm{M}^{-1}$ versus $1.0-2.5 \mathrm{kcal} \mathrm{M}^{-1}$ for H-Y101F; and $\Delta \Delta G=3.9 \pm 1.1 \mathrm{kcal} \mathrm{M}^{-1}$ versus $4 \mathrm{kcal} \mathrm{M}^{-1}$ for H-Y101A (this paper and refs 10 and 12). Thus, the effects of the mutations H-Y101F and H-Y101A on the affinity for lysozyme were the same in the context of the FvD1.3 fragment and scFvD1.3(L-T94ANBD) conjugate.

Properties of a Mixture of Conjugates. The titration curve of a conjugate with lysozyme should allow one to deduce the concentration of lysozyme from a measurement of fluorescence intensity. The relative error $\mathrm{d}[\mathrm{A}]_{0} /[\mathrm{A}]_{0}$ on the concentration of lysozyme $[\mathrm{A}]_{0}$, deduced from such a measurement, was calculated for the scFvD1.3(L-T94ANBD) conjugate and its two mutant derivatives, carrying a Tyr, Phe, or Ala residue in position H-101 (see the Materials and Methods and Figure 5). Each conjugate, taken individually, enabled one to measure the concentration of lysozyme on more than 1 order of magnitude with an error lower than 10\%: $0.06-0.7 \mu \mathrm{M}$ for the wild type, $0.2-8.0 \mu \mathrm{M}$ for the $\mathrm{H}-\mathrm{Y} 101 \mathrm{~F}$ mutant, and $0.7-50 \mu \mathrm{M}$ for the H-Y101A mutant. We observed that the dynamic ranges of the three conjugates were different but overlapping. We therefore made the hypothesis that a mixture of the three conjugates could allow one to measure the concentration of lysozyme with precision on the union of the three individual ranges.

To test this hypothesis, we first titrated an equimolar mixture (500 nM each) of the three derivatives of scFvD1.3(L-T94ANBD) with lysozyme $(0-120 \mu \mathrm{M}$, final concentration). Equation 4.5, giving the fluorescence intensity of a mixture of conjugates as a function of the total concentration in antigen, was fitted to the experimental values with the characteristic parameters of the three conjugates as fitting parameters (see the Materials and Methods and Figure 4). We assumed that the values of $f_{\mathrm{b}}$ and $\Delta f_{\mathrm{c}}$ were identical for the three conjugates. The proportion of active molecules of the conjugate $(85 \pm 8 \%)$, the values of the molar fluorescences $f_{\mathrm{b}}$ and $f_{\mathrm{c}}$, and the values of $K_{\mathrm{D}_{i}}$ were consistent with 
the values obtained in the experiments of individual titration (Table 4). The relative error $\mathrm{d}[\mathrm{A}]_{0} /[\mathrm{A}]_{0}$ on the total concentration $[\mathrm{A}]_{0}$ of lysozyme for the mixture of conjugates showed that this mixture behaved operationally as a single conjugate, enabling one to measure the concentration of lysozyme with a precision of $10 \%$ on a dynamic range covering 3 orders of magnitude (Figure 5).

\section{DISCUSSION}

We have derived a rigorous theory of the signal for fluorescent biosensors, from simple physical laws. In particular, we have established the analytical expressions of their sensitivity $s$, relative sensitivity $s_{\mathrm{r}}$, and lower limit of detection (eqs 3.6-3.8). In the frequent situation where the dissociation constant $K_{\mathrm{D}}$ between a fluorescent biosensor and its antigen is much lower than its total concentration, $s$ is equal to the variation $\Delta f_{\mathrm{c}}$ of its molar intensity of fluorescence on the binding of the antigen and $s_{\mathrm{r}}$ is equal to the relative variation $\Delta f_{\mathrm{c}} / f_{\mathrm{b}}$ of that same intensity (eq 3.9).

We assumed that we could improve the sensitivity $s$ or $s_{\mathrm{r}}$ of fluorescent biosensors by using the additivity of the fluorescence intensities. To test this assumption, we constructed 10 double conjugates between the scFvD1.3 antibody fragment and the IANBD ester fluorophore, from 7 residues that were located at the periphery of its paratope and gave operational single conjugates and from 2 residues that were located far from its paratope. The characterization of the double conjugates revealed fluorescence resonance energy homotransfers (FREHTs) between the two identical fluorescent groups (Table 2). In 5 of 10 cases, we found that the sensitivities $s$ and $s_{\mathrm{r}}$ were lower for the double conjugate than for the best parental single conjugate because of the FREHT phenomenon (Table 3). However, we observed that the efficiency of FREHT was higher in the free state than in the bound state of the conjugates (Table 2). Because of this differential FREHT, the sensitivity $s$ or $s_{\mathrm{r}}$ of 5 double conjugates was improved relative to the best parental single conjugate (Table 3). This improvement was the most visible for the double conjugate at positions (L-Asn31 and L-Ser65); the conjugation of a second fluorescent group in position L-Ser65, far from the paratope, improved the sensitivity $s$ of the conjugate in position L-Asn31 by 3 -fold. Among the 7 operational single conjugates that we studied, 5 could thus be improved at the level of either $s$ or $s_{\mathrm{r}}$ by the coupling of a second fluorophore group (Table 3). The 2 exceptions, scFvD1.3(L-Y49ANBD) and scFvD1.3(L-T94ANBD), corresponded to the 2 single conjugates that had the highest sensitivities (Table 1).

The dynamic range of operational conjugates generally covers more than 1 order of magnitude in concentration (3, 4 ). We showed that this range could be greatly extended by design. We started from the operational conjugate of scFvD1.3 at residue L-Thr94, which is located at the periphery of the paratope, and we introduced the changes $\mathrm{H}-\mathrm{Y} 101 \mathrm{~F}$ and H-Y101A, which affect the center of the paratope and decrease the affinity between FvD1.3 and lysozyme 100- and 1000-fold, respectively. We found that the mutations of residue H-Tyr101 did not affect the fluorescence properties of the scFvD1.3(L-T94ANBD) conjugate (Table 4). The three derivatives of the scFvD1.3(LT94ANBD) conjugate, carrying either residue Tyr, Phe, or
Ala in position $\mathrm{H}-101$, had dynamic ranges that were centered around their $K_{\mathrm{D}}$ value. An equimolar mixture of the three derivatives had a dynamic range covering more than 3 orders of magnitude (Figure 4) and allowed us to titrate the antigen with a relative error lower than $10 \%$ within this range (Figure 5).

Mechanism of Differential FRET. We showed that the transfer of resonance energy between the two fluorescent groups of some conjugates was larger in their free state than in their antigen-bound state; i.e., we observed a differential FRET. The efficiency of transfer $e$ follows the Förster equation

$$
e=C \kappa^{2} Q_{1} Q_{2} r^{-6}
$$

where $C$ depends on the spectral properties of the fluorophores, $\kappa^{2}$ is a scalar depending on the relative orientation of the aromatic rings of the fluorophores and varying from 0 for orthogonal rings to 4 for collinear and parallel rings, $Q_{1}$ and $Q_{2}$ are the quantum yields of the two fluorophores, and $r$ is the distance between them (5). A value of $e_{\mathrm{c}}$, in the bound state, lower than the value of $e_{\mathrm{b}}$, in the free state, for a double conjugate could result from a change either in the average orientation of the two fluorophore rings or in the distance separating them on the binding of lysozyme. The two mechanisms could apply. The aromatic rings could take variable orientations in the free state of the double conjugates (e.g., $\kappa^{2}=2 / 3$ for a full freedom of rotation around three axes) and be immobilized in nearly orthogonal orientations by the binding of lysozyme $(\kappa=0)$. This mechanism is consistent with the observation that the binding of the antigen increases the fluorescence intensity of our conjugates and shields their fluorescent group from the solvent $(3,4)$. The binding of lysozyme could reorientate the two fluorescent groups toward the solvent and thus increase the mean distance between them. This mechanism is consistent with the peripheral location of the fluorescent groups, relative to the paratope, in our conjugates, and their lack of effect on the affinity of the scFv fragment for lysozyme (3).

Use of Differential FRET to Increase Sensitivity. Both mechanisms, described above, could be the cause of the observed increases in $\Delta f_{\mathrm{c}}$ and $s$ when a second fluorophore was coupled to residue L-Ser65, which is beyond the periphery of the paratope. Our results suggest that it could be possible to incrementally improve the sensitivity of a conjugate by coupling several fluorophores in or beyond the periphery of the paratope. The additional coupling sites need not be chosen from the crystal structure of the complex between the antibody and antigen, as described here. They could also be chosen from the amino acid sequence of the Fv fragment alone or from a model of the fragment structure, constructed by homology. The differential FREHT that we observed here with an anti-lysozyme antibody could be applied to antibodies directed against haptens, when the binding of the antigen does not shield the fluorescent group from the solvent enough to result in a measurable variation of fluorescence. The spreading apart of two fluorescent groups or the restriction of their rotational freedom by the binding of a haptenic antigen could increase this variation.

Extending the Dynamic Range with Composite Conjugates. We constructed mutant derivatives of the scFvD1.3(LT94ANBD) conjugate to modify and extend its dynamic 
range, i.e., the interval of concentration in which it was possible to quantify lysozyme with precision. The additional mutations, H-Y101F or H-Y101A, decreased the affinity of the scFvD1.3 conjugate for lysozyme; they were chosen from the structure and mutagenesis data on FvD1.3 (12). A similar approach has been described for a fluorescent conjugate of the maltose-binding protein from $E$. coli. The properties of the maltose-binding protein made it possible to couple the fluorophore at an allosteric site, far from the maltose-binding site, and to independently engineer the two sites (13). In our design, the fluorophore was coupled at position L-Thr94 in the periphery of the antigen-binding site and the additional mutation was introduced at position H-Tyr101 within the binding site. Therefore, our design did not rely on any particular property of the protein receptor, as for the maltosebinding protein. We showed here that the effects of the additional mutations, H-Y101F or H-Y101A, on the affinity for lysozyme were the same in the contexts of the FvD1.3 fragment and scFvD1.3(L-T94ANBD) conjugate and that their effects on the fluorescent properties of the conjugate were negligible. We have previously shown that mutation L-T94C and the coupling of the fluorophore to the mutant Cys residue do not affect the affinity of scFvD1.3 for lysozyme significantly (3). Thus, the recognition of the antigen and the transduction of this recognition event into a measurable signal by the coupled fluorophore behaved as two independent processes in our design.

Does this approach require structural data? Previously, we have developed rules of design to choose the site of fluorophore coupling from sequence and mutagenesis data on the CDR loops of an scFv, without the need for any structural data. Moreover, the conjugates that we designed according to these new rules had the same affinity for the antigen as the parental scFv fragment (4). Thus, simple mutagenesis data should be sufficient to choose the coupling site of the fluorophore and engineer the affinity of the resulting fluorescent conjugate, without interference between the two processes. The methods of in vitro directed evolution could be used to improve the affinity of the parental $\mathrm{scFv}$ fragment (14-16). Therefore, it should be possible to construct a series of conjugate derivatives with affinities covering as many orders of magnitude as needed. A mixture of such conjugate derivatives would behave as a single biosensor, enabling the quantification of an antigen on a very wide interval of concentrations.

Influence of the Fluorometric Setup on Sensitivity. In the Theory, we showed that the sensitivity $s$, the relative sensitivity $s_{\mathrm{r}}$, and the lower limit of detection of the conjugates improve when their total concentration $[\mathrm{B}]_{0}$ and their affinity for the antigen increase. These variations were not involved in our experiments because the value of $[\mathrm{B}]_{0}$ was kept constant, the value of $K_{\mathrm{D}}$ was not affected by the mutations into Cys and the coupling of a fluorophore, and the value of $K_{\mathrm{D}}$ was much lower than [B] $]_{0}$ (eqs 3.6-3.9).

What was the impact of the variations in sensitivity $s$ between conjugates on $\delta[\mathrm{A}]_{0}$, their lower limit of detection? $\delta[\mathrm{A}]_{0}$ is related to the dead-band $\delta F$ of the conjugates by eq 3.8 or, approximately but validly here, by $\delta[\mathrm{A}]_{0}=\delta F / s$ $\approx \delta F / \Delta f_{\mathrm{c}}$ (eq 3.9). Therefore, $\delta[\mathrm{A}]_{0}$ should not depend on the spectrofluorometer and its setup (for example, the factor of photomultiplication) if $\delta F$ was proportional to the fluorescence intensity $F_{0}$ of the free conjugate. In our experimental system, we found that $\delta F$ was independent of $F_{0}$. We evaluated $\delta F$ by comparing the measured and fitted values of $F$ in titrations of single conjugates with lysozyme (this paper and ref 3). The absolute difference between these values varied little along the titration curves. Moreover, their standard deviation, calculated along the titration curves, varied little between conjugates and was equal to $1.7 \pm 0.1$ (mean $\pm \mathrm{SE}$ ). Consequently, an increase in $s$ translated into a proportional decrease of $\delta[\mathrm{A}]_{0}$. The constancy of $\delta F$ could have two causes. First, the manipulations of the spectrofluorometer cuvette could introduce larger perturbations on the measurements than the optical setup. Second, the measured values of $F$ belonged to a small subsegment of the full range of the instrument, in which $\delta F$ was practically constant.

However, the dead-band $\delta F$ of our immunosensors might depend on the fluorescence intensity $F_{0}$ of their free state in other experimental conditions, for example, if they were immobilized $(17,18)$. In these cases, the dead-band $\delta F$ could be expressed as a percentage of the fluorescence signal $F_{0}$ and the lower limit of detection $\delta[\mathrm{A}]_{0}$ could be expressed as a percentage of the total concentration in immunosensor $[\mathrm{B}]_{0}$, with the two parameters being related by the relative sensitivity $s_{\mathrm{r}}=\Delta f_{\mathrm{c}} / f_{\mathrm{b}}$ (eqs 3.4 and 3.9 ). Therefore, the coupling of a second fluorophore, as described here, could also decrease the lower limit of detection in these cases.

Affinity and Kinetics Constraints for Continuous Measurements. In principle, a reagentless fluorescent biosensor could measure the concentration of an antigen continuously, for example, in the blood stream $(19,20)$ or within a single living cell (18). For such measurements, the rate constants of association $k_{\text {on }}$ and dissociation $k_{\text {off }}$ between the biosensor and ligand must be faster than the rate with which the concentration of the ligand varies. In many instances, the affinity will not be a limiting parameter. For example, the concentrations of many hormones in the blood stream are in a nanomolar range; a total of 100 copies of a protein in a spherical cell of $1 \mu \mathrm{m}$ radius gives a concentration of a few nanomolars. Such concentrations are measurable at equilibrium with our present setup. However, the high affinities between the antibodies and antigens result from very low $k_{\text {off }}$ values, which constitute an obstacle to their use for continuous measurements (21). Point mutations in the paratope of an antibody generally decrease affinity by increasing $k_{\text {off. In particular, such is the case for mutations }}$ H-Y101F and H-Y101A of mAbD1.3 that we used here (12). The engineering of affinity could therefore make possible continuous measurements but at the expense of sensitivity, as shown by eqs $3.6-3.8$. A solution to this problem would be to compensate for the increase in $k_{\text {off }}$ by a parallel increase in $k_{\text {on }}$ through existing methods, to the point where $k_{\text {on }}$ itself will be limited by the diffusion rate of molecules $(22,23)$.

\section{CONCLUSION}

We found that the coupling of two fluorescent groups of IANBD ester to the same molecule of $\mathrm{scFv}$ resulted in a FREHT phenomenon. The efficiency of FREHT was more important in the free state of the double conjugate than in its bound state. This differential FREHT could result from a change on the binding of the antigen, either in the average orientation of the two fluorophore rings or in the distance 
separating them. It enabled us to increase the sensitivity of a conjugate by coupling a second fluorescent group to a residue located far from the paratope. These findings will be useful to improve the sensitivity of fluorescent immunosensors by design and extend their construction to antibodies directed against haptens. Moreover, our approach should help to dissect and better understand the mechanisms of fluorescence energy transfer within proteins. We showed that the dynamic range of a fluorescent immunosensor could be greatly widened by design, and this possibility will broaden their utility. The knowledge that is necessary to design and improve reagentless fluorescent biosensors from antibodies consists of sequence and mutagenesis data and could be generated at high throughput. Our approach could be extended to ligand-binding proteins other than antibodies and ultimately contribute to generate a set of highly specific, sensitive, and quantitative analytical tools against the full complement of the human proteome, with myriads of applications.

\section{ACKNOWLEDGMENT}

We thank Shamila Naïr for critical reading of the manuscript.

\section{REFERENCES}

1. Branden, C., and Tooze, J. (1999) Introduction to Protein Structure, 2nd ed., Chapter 13, Garland Publishing Inc., New York.

2. Thevenot, D. R., Toth, K., Durst, R. A., and Wilson, G. S. (2001) Electrochemical biosensors: Recommended definitions and classification, Biosens. Bioelectron. 16, 121-131.

3. Renard, M., Belkadi, L., Hugo, N., England, P., Altschuh, D., and Bedouelle, H. (2002) Knowledge-based design of reagentless fluorescent biosensors from recombinant antibodies, J. Mol. Biol. 318, 429-442.

4. Renard, M., Belkadi, L., and Bedouelle, H. (2003) Deriving topological constraints from functional data for the design of reagentless fluorescent immunosensors, J. Mol. Biol. 326, 167175.

5. Lakowicz, J. R. (1999) Principles of Fluorescence Spectroscopy, 2nd ed., Kluwer Academic/Plenum Publishers, New York.

6. Kunkel, T. A., Roberts, J. D., and Zakour, R. A. (1987) Rapid and efficient site-specific mutagenesis without phenotypic selection, Methods Enzymol. 154, 367-382.

7. Pace, C. N., Vajdos, F., Fee, L., Grimsley, G., and Gray, T. (1995) How to measure and predict the molar absorption coefficient of a protein, Protein Sci. 4, 2411-2423.

8. Wolfram, S. (1996) The Mathematica Book, 3rd ed., Wolfram Media/Cambridge University Press, U.K.
9. Bhat, T. N., Bentley, G. A., Boulot, G., Greene, M. I., Tello, D., Dall'Acqua, W., Souchon, H., Schwarz, F. P., Mariuzza, R. A., and Poljak, R. J. (1994) Bound water molecules and conformational stabilization help mediate an antigen-antibody association, Proc. Natl. Acad. Sci. U.S.A. 91, 1089-1093.

10. Hawkins, R. E., Russell, S. J., Baier, M., and Winter, G. (1993) The contribution of contact and non-contact residues of antibody in the affinity of binding to antigen. The interaction of mutant D1.3 antibodies with lysozyme, J. Mol. Biol. 234, 958-964.

11. Dall'Acqua, W., Goldman, E. R., Eisenstein, E., and Mariuzza, R. A. (1996) A mutational analysis of the binding of two different proteins to the same antibody, Biochemistry 35, 9967-9976.

12. England, P., Bregegere, F., and Bedouelle, H. (1997) Energetic and kinetic contributions of contact residues of antibody D1.3 in the interaction with lysozyme, Biochemistry 36, 164-172.

13. Marvin, J. S., Corcoran, E. E., Hattangadi, N. A., Zhang, J. V., Gere, S. A., and Hellinga, H. W. (1997) The rational design of allosteric interactions in a monomeric protein and its applications to the construction of biosensors, Proc. Natl. Acad. Sci. U.S.A. 94, 4366-4371.

14. Low, N. M., Holliger, P., and Winter, G. (1996) Mimicking somatic hypermutation: Affinity maturation of antibodies displayed on bacteriophage using a bacterial mutator strain, J. Mol. Biol. 260, 359-368.

15. Schaffitzel, C., Hanes, J., Jermutus, L., and Pluckthun, A. (1999) Ribosome display: An in vitro method for selection and evolution of antibodies from libraries, J. Immunol. Methods 231, 119-135.

16. Hanes, J., Schaffitzel, C., Knappik, A., and Pluckthun, A. (2000) Picomolar affinity antibodies from a fully synthetic naive library selected and evolved by ribosome display, Nat. Biotechnol. 18 , 1287-1292.

17. Ligler, F. S., Breimer, M., Golden, J. P., Nivens, D. A., Dodson, J. P., Green, T. M., Haders, D. P., and Sadik, O. A. (2002) Integrating waveguide biosensor, Anal. Chem. 74, 713-719.

18. Vo-Dinh, T., Alarie, J. P., Cullum, B. M., and Griffin, G. D. (2000) Antibody-based nanoprobe for measurement of a fluorescent analyte in a single cell, Nat. Biotechnol. 18, 764-767.

19. Peterson, J. I., and Vurek, G. G. (1984) Fiber-optic sensors for biomedical applications, Science 224, 123-127.

20. Pickup, J. C. (1985) Biosensors-A clinical perspective, Lancet 2 (8459), 817-820.

21. Morgan, C. L., Newman, D. J., and Price, C. P. (1996) Immunosensors: Technology and opportunities in laboratory medicine, Clin. Chem. 42, 193-209.

22. Schreiber, G., and Fersht, A. R. (1996) Rapid, electrostatically assisted association of proteins, Nat. Struct. Biol. 3, 427-431.

23. Selzer, T., Albeck, S., and Schreiber, G. (2000) Rational design of faster associating and tighter binding protein complexes, Nat. Struct. Biol. 7, 537-541.

24. Bernstein, H. J. (2000) Recent changes to RasMol, recombining the variants, Trends Biochem. Sci. 25, 453-455.

BI048922S 